\title{
The Hittite Notion of Border. A practical view
}

The Hittite cuneiform texts (16th-12th cent. BCE) contain a number of detailed descriptions of borders of various countries and territories. Such fragments, mainly found in the international treaties as well as the annals of the kings, clearly reflect the particular formal interest on the part of the Hittite rulers in specific delimitation of political territories.

Although the Hittite listings of the topographical points, which make up a frontier can extend over many lines of texts, one never finds any use of cardinal directions which would indicate the position of these points. Instead, the Hittite scribes applied a peculiar internal reference system that combines the position of the speaker and another point of reference in order to locate a specific part of the border.

The talk will briefly review the corpus of the Hittite border descriptions and discuss how such fragments were conceptualized. It will also tackle the question, whether they reflect a 'bird's eye', cartographic view of the land, or rather a ground-level, hodological perspective. 